\title{
Photocatalysis: fundamentals and applications in JEP 2011
}

\author{
Sylvie Lacombe $\cdot$ Nicolas Keller
}

Received: 31 May 2012 / Accepted: 12 June 2012 /Published online: 18 July 2012

(C) Springer-Verlag 2012

As part of catalysis - and more precisely of heterogeneous catalysis-heterogeneous photocatalysis is an area of chemistry impacting many reactions as varied as total or mild oxidation reactions, dehydrogenation reactions, metal deposition, hydrogen transfers, etc. These different reactions are mainly facing applications in the field of water and air purification treatments - targeting both chemical pollutants and biological ones, self-decontaminating or self-cleaning products, organic fine chemistry as well as energy-related areas with hydrogen production from water.

Relative to the history of catalysis processes, coined by Berzelius at the beginning of nineteenth century, photocatalysis remains a recent discipline, as marked by J.M. Herrmann in its introductory paper of this Special Issue. Basically, photocatalysis differentiates from conventional catalysis by the activation of the catalytic solid, which occurs through photon absorption rather than by thermal activation. This

\footnotetext{
S. Lacombe $(\bowtie)$

Institute for Analytical Sciences and Physico-Chemistry for Environment and Materials (IPREM), UMR 5254 CNRS, University of Pau and Pays de l'Adour,

Hélioparc : 2 avenue of President ANGOT, 64053 Pau Cedex 09, France

e-mail: sylvie.lacombe@univ-pau.fr

\section{N. Keller}

Laboratory of Materials, Surfaces and Processes for Catalysis (LMSPC), European Chemistry School, Polymers and Materials (ECPM), UMR 7515 CNRS, University of Strasbourg,

25 rue Becquerel,

67087 Strasbourg Cedex, France

e-mail: nkeller@unistra.fr
}

photonic activation thus requires the use of a semiconductor material as catalyst, provided that the radiation wavelengths are greater than its band gap, which corresponds to the energy gap between both conduction and valence bands of the semiconductor.

Activating a semiconductor leads to the promotion of an electron from the valence to the conduction band, with the simultaneous creation of a photogenerated hole within the valence band. Further, the transfer of photogenerated charge carriers to the photocatalyst surface allows redox reactions to occur with adsorbed reactants, coming from gas or liquid phase depending on the application. At the catalyst surface, the redox reactions are separated into reduction and oxidative steps, involving on one hand, conduction band electrons and adsorbed electron acceptors such as, e.g., oxygen molecules $\left(\mathrm{e}_{\mathrm{CB}}{ }^{-}+\mathrm{A} \rightarrow \mathrm{A}^{--}\right)$, and on the other hand, valence band holes and adsorbed electron donors such as, e.g., organic molecules or more generally the targeted pollutant $\left(\mathrm{h}_{\mathrm{VB}}{ }^{+}+\right.$ $\mathrm{D} \rightarrow \mathrm{D}^{\bullet+}$ ). Indirect oxidation reactions also occur through the formation of highly oxidative hydroxyl radicals generated by the oxidation of water by holes. Thus, the photocatalysis discipline exists through the ability of a material, in this case a semiconductor (usually $\mathrm{TiO}_{2}$ ), to simultaneously interact with light and reactants, through both ab- and adsorption phenomena, respectively.

Nowadays, the "Advanced Oxidation Process" (AOP) nature of photocatalysis remains the most exploited, facing applications in the field of water and air purification treatments and self-decontaminating/self-cleaning products. On one hand, the implementation of photocatalysis for environmentally friendly cleaning water or air fits into a sustainable development approach, driven by the search for alternative 
technologies meeting the requirements of both sustainable chemistry and technological processes. In this context, air purification remains till now more developed than water detoxification, for purifying industrial effluents as well as indoor air in confined rooms. On the other hand, selfdecontaminating/self-cleaning surface applications can concern materials and products as varied as glass, cements, concretes, tiles, ceramics, paints, textiles, etc. for which the photocatalytic function is seen as an added value for the product use and commercialization. It is also worth noting that all the AOP applications of photocatalysis can target the oxidative mineralization of hazardous, environmentally unfriendly, or even simply unpleasant odorous chemical molecules, as well as a very large variety of microorganisms, seen as a complex assembly of high molecular weight compounds (bacteria, virus, spores, etc.).

The main markets forecasted for photocatalysis and photocatalyst-based products are the construction, consumers, automotive, environment, energy, and health market sectors (Fig. 1). The largest part accounting for $75 \%$ of the global market and acting in the coming years as driven force for the implementation of the technology will still worldwide concern the construction sector with both outdoor and indoor products (self-cleaning glass, cement, concrete, paintings, etc.), among which one of the most significant current and future market is the de-NOx outdoor air treatment (building walls, tunnels, roofs, roads, etc.). The BCC study analyzed that the estimated global market has strongly increased starting from 2005 to reach $€ 1,500$ millions in 2015. It is also worth nothing that, even if Asia is actually - and in the coming years will remain - the major market with $60 \%$ of the global revenues, the European market will comfort its second ranking, with a yearly $20 \%$ progression, well ahead of the American market.

During the last four decades that constitute the history of photocatalysis, this discipline benefited from many skills and know-how arising from research fields as diverse as catalysis, photochemistry, electrochemistry, surface science, chemical engineering, analytical chemistry, solid state science, etc. This diversity of approaches helped in both growth and enlargement of the photocatalysis community, and can now be seen as one of the wealth of the discipline.

Within this multidisciplinary approach and context, the European Photocatalysis Federation (EPF; http:// www.photocatalysis-federation.eu/) is a newborn association (2010) with more than 97 European members (companies, universities, technical centers, and research laboratories) aimed at the development of the photocatalysis market in Europe. EPF is currently organized in four Local Committees, namely the UK Semiconductor Photochemistry Network (Photocatalysis section), the German Federation for Applied

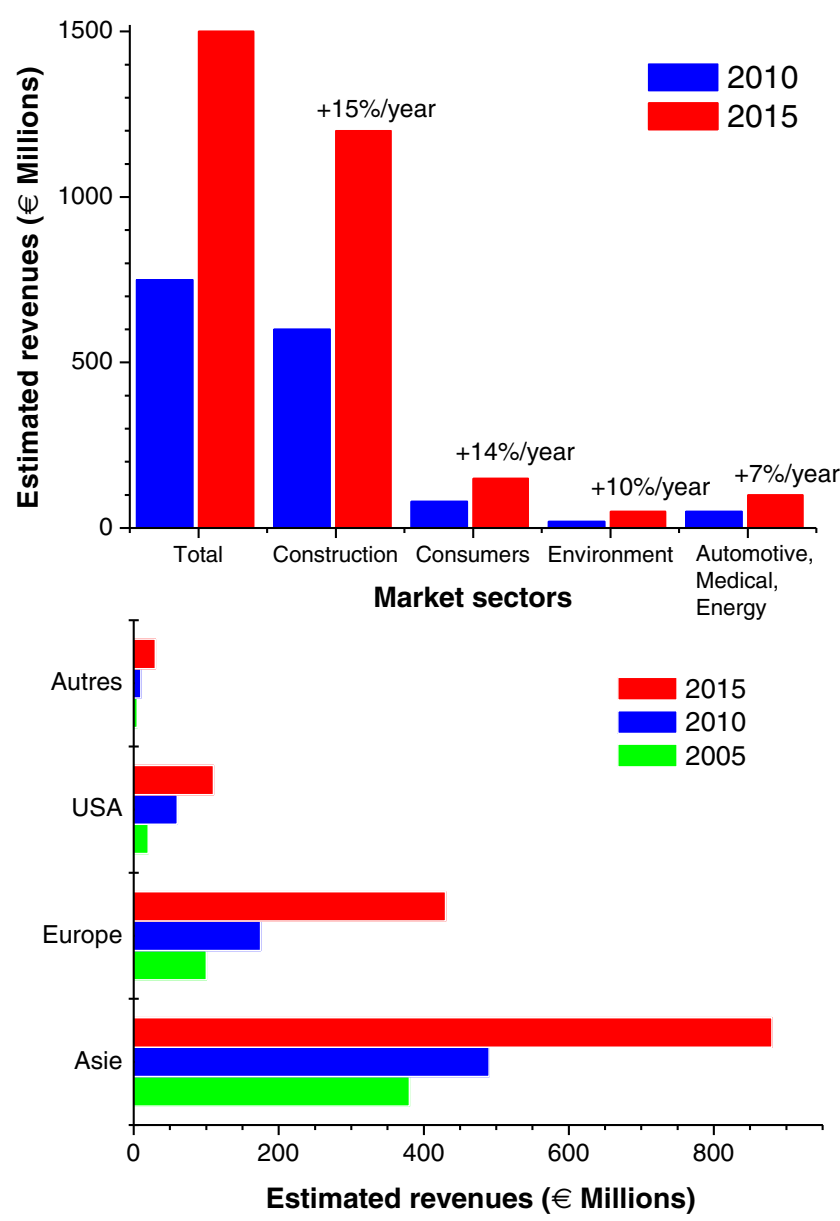

Fig. 1 Market sectors and geographical markets for photocatalysis (from BCC Research, March 2010 high tech analysis, data published on behalf of the European Photocatalysis Federation)

Photocatalysis, the Iberic Association for Photocatalysis, and the French Photocatalysis Federation.

EPF organized in Bordeaux (France) the two first editions of Journées Européennes de la Photocatalyse/European Symposium on Photocatalysis (JEP), JEP 2009 and JEP 2011, in collaboration with Innovalis Aquitaine and University of Pau and Pays de l'Adour. JEP 2011 gathered 250 attendees from 31 countries, most of them from all over Europe (91\%). Scheme 1 below indicates that if many attendees came from universities, research laboratories, or technical centers, a significant contribution from companies was observed (24\%). JEP 2011 thus showed a significant improvement relative to the previous JEP 2009 with 150 attendees. Besides the 6 invited conferences, 17 oral presentations together with the intensive and long-lasting poster session (119 posters exhibition) of JEP 2011, the 64 bilateral meetings scheduled by Enterprise Europe South-West France between attendees who wanted to start new relationships were one of the main successes of JEP 2011. The success of JEP 2011 highlights the 


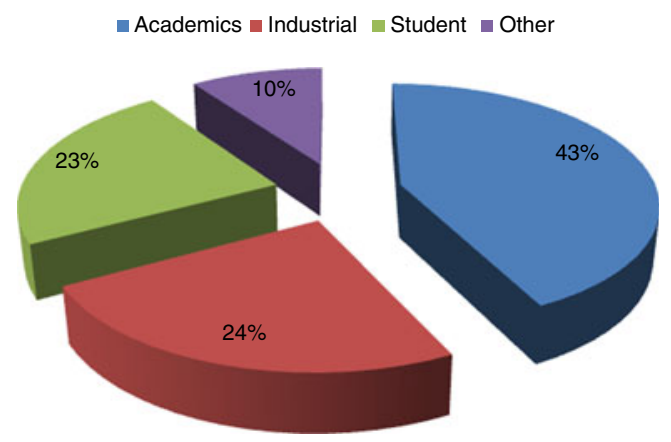

Scheme 1 Attendees distribution to JEP 2011 in Bordeaux

potential of photocatalysis as an emerging AOP for the treatment of chemical pollutants or of microorganisms in air or in water, and for the development of self-cleaning materials.

This special issue is a collection of 15 key articles selected from the scientific presentations of JEP 2011. The paper of Herrmann lays the basis of photocatalysis and recalls the fundamental principles and definitions, with reference to the often used photocatalyst, titanium dioxide $\left(\mathrm{TiO}_{2}\right)$. The following paper by Emeline et al. describes the way to secondand third-generation photocatalysts. The tendency to decrease the energy of the band gap to extend the spectral sensitivity to visible light in second-generation photocatalysts is completely opposite the fundamental observation in first-generation photocatalysts, whereby the activity increased with increasing band gap energy. Hence, some notions are reconsidered to produce third-generation photoactive materials.

Other papers illustrate the intense research devoted to elaboration and properties of new photocatalytic materials in order to increase their efficiency, particularly under visible light. The paper from the team of Faria reviews some important aspects of graphene-based $\mathrm{TiO}_{2}$ composite photocatalysts. The paper from Bahneman's group deals with the compared photocatalytic efficiency of transition metalcontaining zinc oxides under UV and visible light, with a noticeable increased activity of Ru-modified zinc oxide relative to titanium dioxide-based catalysts under visible light. Michalow et al. describe niobium doping of $\mathrm{TiO}_{2}$, affecting its structural, optical, and photocatalytic properties and enhancing its photocatalytic performance under UVA and vis irradiation at low dopant level. Pausova et al. have studied the influence of $\mathrm{TiO}_{2}$ nanoparticles' immobilization on the surface of $\mathrm{Mg}_{2} \mathrm{Al}-\mathrm{LDH}$ nanosheets on the degradation of model pollutants in water. The work from Trapalis's team investigates the influence of fluorine ion during the solvothermal synthesis of anatase $\mathrm{TiO}_{2}$ on the crystallinity and performances for NO oxidation.
Another hot topic in the field of environmental applications concerns the immobilization of the photocatalytic coating onto various supports for an easier handling and recovering of the catalyst. In this respect, the alveolar $\mathrm{TiO}_{2} / \mathrm{SiC}$ foams described and used for water treatment by Keller's team represent a significant improvement. For gasphase experiments, the team of Lavrencic-Stangar used nontransparent highly photocatalytically active coatings based on a silica/titania binder and homogeneously dispersed $\mathrm{TiO}_{2}$ powders on $\mathrm{Al}$ sheets. The adsorption properties and photocatalytic activity of immobilized photocatalysts were very effective and exhibited a considerably improved adhesion and uniformity.

A lot of studies are also devoted to the improvement of photocatalytic oxidation efficiency by properly designing suitable reactors or systems. An original approach from Nae-Lih Wu's team based on $\mathrm{TiO}_{2}$ particle deposition on the surface of disks was used in a multilayer rotating disk reactor for the elimination of a dye, methyl orange, in aqueous solution. $\mathrm{TiO}_{2}$ thin films prepared by PVD magnetron sputtering on an electrode proved to be efficient for in situ discoloration of indigo carmine dye in a flow microreactor under UV light and electric field. This system clearly demonstrates the integration of photoelectrochemical processes for enhancing water decontamination, as reported by Montero-Ocampo et al.

The important issue of the photocatalytic test validity is addressed in two papers. The first one by Kartheuser et al. is related to the standardization effort in the field of photocatalysis in Europe. This paper describes a proposed standardized test at the CEN level to determine the mineralization efficiency of photocatalytic media and systems (air cleaners) against a selected mixture of VOCs in a closed chamber. The second paper by Simonsen et al. correlates various photoactivity tests (dye bleaching, stearic acid degradation, and gaseous acetone mineralization) of commercial $\mathrm{TiO}_{2}$ coatings and provides promising results for future work on standardization.

Two papers are devoted to microorganism deactivation. The photoelectrocatalytic oxidation using reference strains of Enterococcus faecalis and Escherichia coli as fecal indicators is evaluated by Venieri et al. for the disinfection of water and wastewater, evidencing the improvement brought by the applied potential on the results. Lastly, the inactivation of bacteria under visible light and in the dark by $\mathrm{Cu}$ polyester thin sputtered layers on textile fabrics is presented by Pulgarin/Kiwi's team.

Authors and reviewers are greatly acknowledged for their contributions. Dr. Philippe Garrigues, responsible editor, is sincerely thanked for the kind invitation to publish this Issue. 


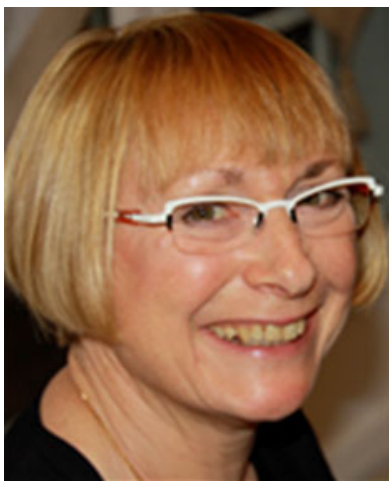

Sylvie Lacombe is CNRS research director in the Institute for Analytical Sciences and Physico-Chemistry for Environment and Materials (IPREM), a mixed CNRS-UPPA (University of Pau and Pays de l'Adour) team. She has been working for 20 years in the photochemistry and photocatalysis field and was interested with sol-gel chemistry for the synthesis of photosensitizers containing hybrid materials or modified $\mathrm{TiO}_{2}$ and with their application for the oxygenation/ mineralization of organic compounds. Current research topics are devoted to reaction mechanisms in heterogeneous photocatalytic/photosensitized reactions. She is also interested with normalization in photocatalysis.

She is in charge of the Photochemistry group in Pau and she is vicepresident of the European Photocatalysis Federation http://www. photocatalysis-federation.eu/.

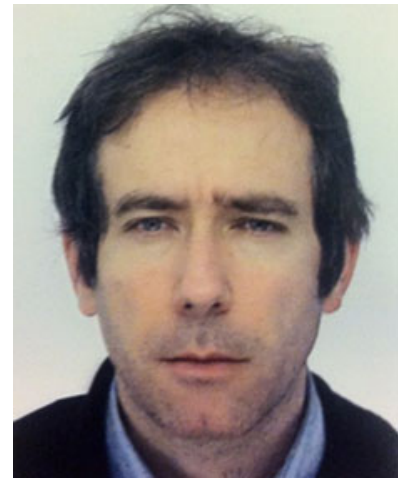

Nicolas Keller is Chargé de Recherches CNRS at the Laboratory of Materials, Surfaces and Processes for Catalysis (LMSPC), a CNRS-Strasbourg University joined unit. He received his $\mathrm{PhD}$ in catalysis and material chemistry in 1999 from the Strasbourg University. After a post-doctoral position at the Fritz-Haber-Institut of the MPG in Berlin, he was appointed in 2001 by the CNRS. He authored 90 scientific papers, 79 talks and 12 patents. He acted as guest-editor for special issues of Catalysis Today and co-organized a European Material Research Society symposium. His current research focus on the use of nano to macro-structured $\mathrm{TiO}_{2}$-based materials for photocatalysis and photoconversion, with applications to environment (air and water detoxification), functionalized textiles, energy (hydrogen production) and health (artificial retina), within a team awarded the 2007 Alsace Regional award for innovation from French national institute of industrial property. 